\title{
Research on the Algorithms of Lane Recognition based on Machine Vision
}

\author{
Minghua Niu ${ }^{1 *}$, Jianmin Zhang, Gen Li \\ ${ }^{1}$ Tianjin University of Technology and Education, \\ Tianjin 300222, China \\ *Corresponding author’s Email:18812616385@163.com
}

\begin{abstract}
Lane recognition is a kind of technology which can reconstruct either continuous or discontinuous lanes in the driving region into the continuous curves from the images of the road containing the lane markings. It is the basis and prerequisite for the whole lane departure warning system and the key technology in intelligent traffic safety assistant driving now. Generally speaking, the road images which the camera captures are processed by three steps: image preprocessing, image threshold segment and lane recognition. This paper proposed an efficient system to detect the lanes and mainly made experimental research on the images simulation of the three kinds of algorithms of the threshold segment and used the principle of Hough Transform in the lane recognition. Finally, the relatively efficient and accurate lane recognition processing algorithm was obtained according to the experimental results. Undoubtedly, this would lay a theoretical foundation for putting lane detection algorithm into practical application.
\end{abstract}

Keywords: Threshold segmentation; OTSU algorithm; Hough transform; Lane recognition

\section{Introduction}

After more than a centurys development, automobile industry has developed rapidly because of its unique superiority. The number of cars is increasing in a straight line. Although people enjoy the convenience of the automobiles, we are also facing serious traffic problems and traffic accidents occurring frequently at the same time. In recent years, automatic detection of lane-departure has attracted much attention because many traffic accident fatalities are related to unintended lane-departures [1]. Countries all over the world are actively researching and developing the vehicle safety assistant. Lane detection and recognition based on machine vision is one of the key techniques for intelligent vehicle driving. It is the basis to implement the LDW [2] (lane departure warning) system and lane keeping of active safety functions. There are already some successful examples of the system for lane recognition, including LOIS, GOLD, RALPH, etc. At present, many lane recognition methods have been proposed. Some simple al- gorithms early for the detection of lane lines mainly use obvious and clear color of lanes, and this often makes error identification; other complex algorithms later have strong anti-interference ability, but the realtime performance is poor. Therefore, how to detec$\mathrm{t}$ the lane lines accurately and rapidly has become a challenge in intelligent vehicle vision navigation.

This paper took the lane-ray images as the research objects. Figure 1 illustrates the basic procedure of the treatment process of road images.

The reminder of this paper is structured as follows. In Section 2, the pretreatment processing of the road image is present. The three algorithms of the threshold segment of the images are described in Section 3. Section 4 does the research about the recognition of the lane lines through the HT (Hough Transform) algorithm. Experimental results are shown and discussed in Section 5. Section 6 concludes the paper. 


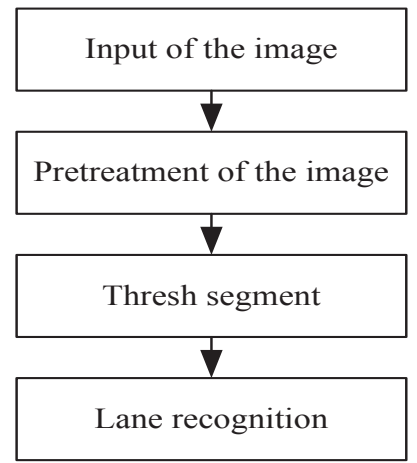

Figure 1 Image processing.

\section{The Pretreatment of Road Images}

Due to the impact of external factors, the images taken by a CCD camera mounted on a test vehicle do not fully reflect all the information of the original images. Therefore, the images should be pretreated firstly to improve their quality, extract the interesting parts and inhibit the useless information.

For the road images that captured by machine vision (on-board camera), preprocessing steps of the image are generally divided into: graying and filtering.

\subsection{Graying the road image}

The images taken by a CCD camera are the RGB color images in the lane line detection and recognition. The RGB model is based on the red (Red), green (Green), blue (Blue) three color brightness variation and the superposition between them to get a variety of colors. Color images contain amount of information and its processing speed is low. So the color image must be converted into gray image firstly. Although the gray image will lose some information inevitably, the information that the gray image includes can separate the lane marking lines from the background enough. Therefore, in order to improve the real-time performance of the system, the color image is converted into the gray image first.

Because the human eye is sensitive to green, the weighted average algorithm by RGB three components can get a reasonable gray image. Figure 2 shows examples of graying the color images of the road, which illustrates the performance of the graying by the weighted average algorithm. Figure 2(b) and Figure 2(d) are the gray images which are converted from Figure 2(a) and Figure 2(c) (the original color images).

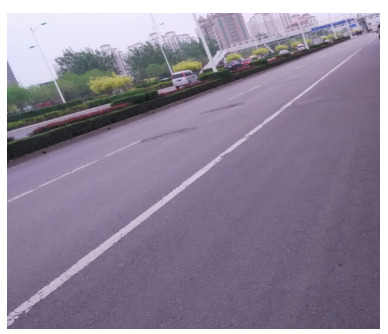

(a) Color image

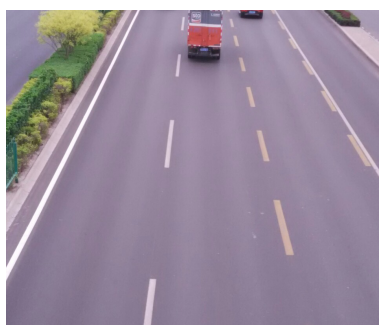

(c) Color image

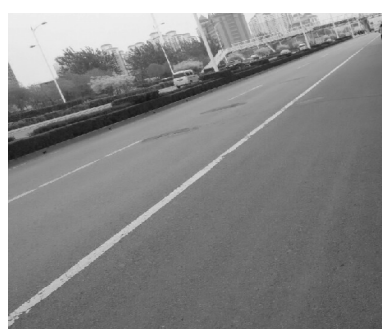

(b) Gray image

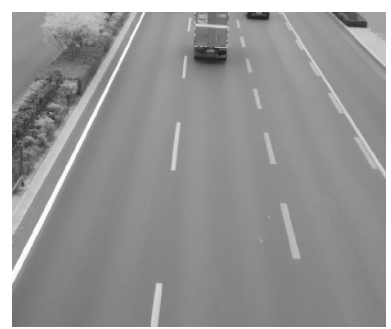

(d) Gray image
Figure 2 Graying the images of the road.

\subsection{Enhance the image}

The main purpose of image enhancement is to reduce the noise of images, and it is necessary for the detection of the lanes. Each original image actually obtains noise more or less. In this paper, the median filtering algorithm was used to filter the image.

Figure 3 shows examples of the median filtering algorithm. The salt and pepper noise was added into the Figure 2(b) and Figure 2(d). Figure 3(a) and Figure 3(c) are the images which are added with the salt and pepper noise. As shown in Figure 3(b)and Figure $3(\mathrm{~d})$, the salt and pepper noise in images is removed, which displays that the median filtering algorithm has good effect.

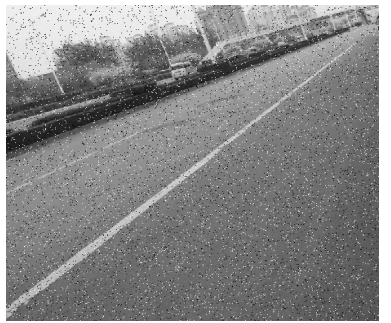

(a) Salt and pepper noise

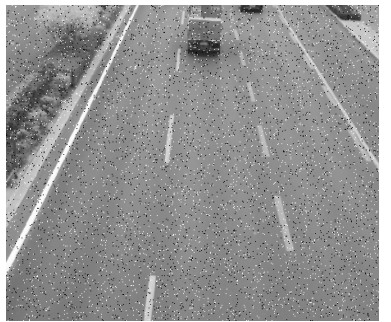

(c) Salt and pepper noise

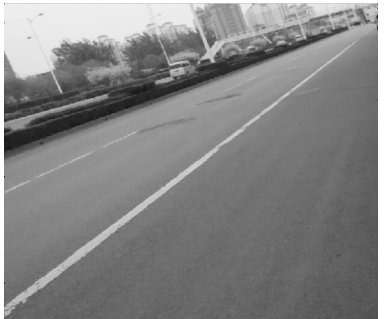

(b) Median filtering

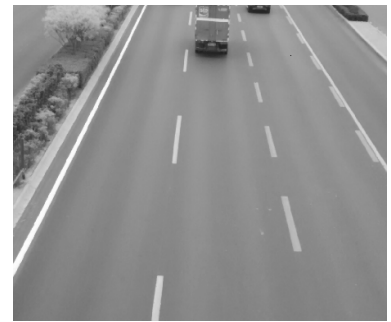

(d) Median filtering

Figure 3 Graying the images of the road. 


\section{Threshold Segmentation of Road Image}

Image segmentation is a key technique of image processing [3] and machine vision field. This paper mainly used threshold value segmentation method to process the road gray image. It made the choice for a threshold, and the original image was converted into black and white two value image. The function for processing the gray threshold segmentation method is shown in equation (1):

$$
g(x, y)= \begin{cases}0, & 0 \leq f(x, y) \leq T \\ 255, & T \leq f(x, y) \leq 255\end{cases}
$$

Among which, $f(x, y)$ is the original image pixel value, then $g(x, y)$ is the pixel value after processing. Gray-level threshold segmentation method is a nonlinear operation, and its function is shown in Figure 4.

This paper studied the automatic threshold segment in the threshold segment of images. Generally, selecting the threshold value automatically can reach stable and reliable effects. In this paper, three algorithms for the threshold segment of the gray images were analyzed.

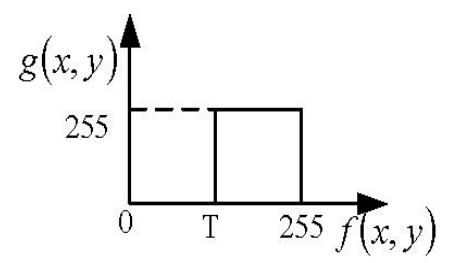

Figure 4 The function of threshold segment.

\subsection{Iteration threshold segmentation}

The basic idea of iteration threshold segmentation is choosing a threshold as the initial estimate value firstly, and then according to certain rules to update the estimate value continuously, until it satisfies the conditions. The iteration algorithm is:

Step1: Choose an initial estimate value $T$.

Step2: Use T divide the image into two regions: $R_{1}$ and $R_{2}$.

Step3: Calculate the average gray value $\mu_{1}$ and $\mu_{1}$ for the region $R_{1}$ and $R_{2}$.

Step4: Calculate the new threshold:

$$
T=\frac{1}{2}\left(\mu_{1}+\mu_{2}\right)
$$

Step5: Repeat the step $2 \sim 4$.

\subsection{The OTSU algorithm}

This method has simple and fast features in processing the image. The thought is: Designing the number of pixels whose gray level is $i$ as $n_{i}$ and the gray scale range is $[0, L-1]$, so the total pixels:

$$
N=\sum_{i=0}^{L-1} n_{i}
$$

The probabilities of every gray value:

$$
p_{i}=\frac{n_{i}}{N}\left(\sum_{i=0}^{L-1} p_{i}=1\right)
$$

Use the threshold value $T$ to divide the pixels into two categories $C_{0}$ and $C_{1} . C_{0}$ is made by the pixels whose gray value range is $[0, T-1]$. And $C_{1}$ is made by the pixels whose gray value range is $[T, L-1]$. The probabilities of the region $C_{0}$ and $C_{1}$ is:

$$
\begin{gathered}
P_{0}=\sum_{i=0}^{T-1} p_{i} \\
P_{1}=\sum_{i=T}^{L-1} p_{i}=1-P_{0}
\end{gathered}
$$

The average gray value of the region $C_{0}$ and $C_{1}$ is:

$$
\mu_{0}=\frac{1}{P_{0}} \sum_{i=0}^{T-1} i p_{i}=\frac{\mu(T)}{P_{0}}
$$

$$
\mu_{1}=\frac{1}{P_{1}} \sum_{i=T}^{L-1} i p_{i}=\frac{\mu-\mu(T)}{1-P_{0}}
$$

The total variances of two areas are:

$\sigma_{B}^{2}=P_{0}\left(\mu_{0}-\mu\right)^{2}+P_{1}\left(\mu_{1}-\mu\right)^{2}=P_{0} P_{1}\left(\mu_{0}-\mu_{1}\right)^{2}$

Let $T$ choose the values in turn in the range $[0, L-1]$, the $T$ value which makes $\sigma_{B}^{2}$ to be the maximum value is the best threshold. So, this method is defined as the OTSU algorithm.

\subsection{Watershed algorithm}

The watershed algorithm is a reference segmentation method of morphological theory. An image is viewed as a topological topographic map. The gray values $f(x, y)$ correspond to terrain height value. High gray value corresponds to the peak. Low gray value corresponds to the valley. The correspond to terrain height value. High gray value corresponds 


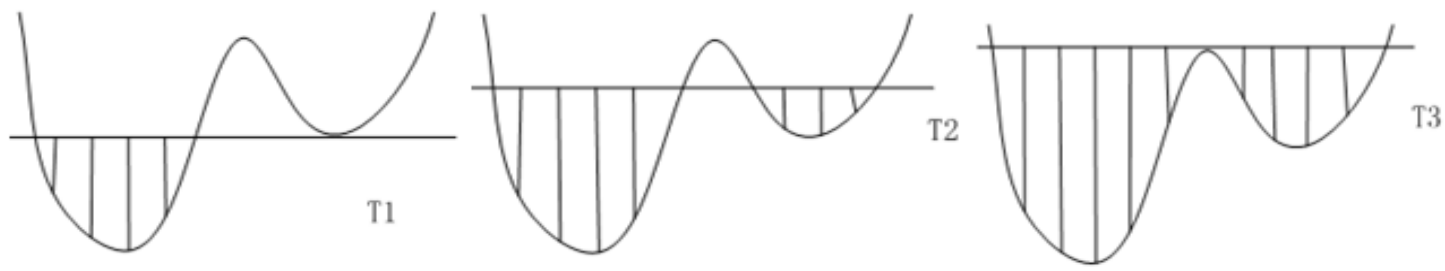

Figure 5 The schematic diagram of watershed segmentation.

to the peak. Low gray value corresponds to the valley. The water is always flowing to low areas, stopping at the local low-lying areas. The low-lying place is called water basin. Finally all the water will be divided into poly in different water absorbent basin. The ridge between basins is known as the watershed. Water flows from the watershed and the possibility of it flows toward different water basin is equal. This idea is applied to image segmentation to find out the different water basin and watershed in the gray image. These different water basins and watershed areas for$\mathrm{m}$ the areas are the targets which we want to segment. Figure 5 illustrates its schematic diagram.

\section{Lane Recognition Based on Hough Trans- form}

Many studies of lane marking detection have been carried out, some of which using RANSAC [4, 6] or the Hough transform. In this paper, the Hough transform was used to detect the lane markings. Hough transform is an important method to detect the shape of discontinuous boundary. The HT extracts the candidates of the lane-related parameters [7]. It transforms the image coordinate space into the parameter space to achieve the fitting of straight line and curve. This paper mainly studied the straight lane recognition.

The equation (10) in polar coordinates indicates a line:

$$
\rho=x \cos \theta+y \sin \theta
$$

where $\rho$ represents the vertical distance from straight line to the origin. $\theta$ represents the angle from $x$ axis to the vertical. The range of $\theta$ is $\left[-90^{\circ},+90^{\circ}\right]$. The schematic of coordinate is shown as Figure 6.

Similar to Cartesian coordinates, Hough Transfor$\mathrm{m}$ in polar coordinates will also transform points in the image coordinates into the parameter space. Expressed in polar coordinates, the collinear points in image coordinates space which is transformed into the parameter space also intersect at the same point. Unlike the Cartesian coordinate, collinear two points $\left(x_{i}, y_{i}\right)$ and $\left(x_{j}, y_{j}\right)$ in image coordinate space which are mapped into the parameter space are two sinusoidal curves expressed in polar coordinates. They intersect at a point as Figure 7.

\section{Experimental Results}

In order to compare the three kinds of algorithm$s$ of the threshold segment of the road images, some captured images were taken as samples. These images were taken by a CCD camera in the normal traffic condition. Figure 8 is an example to prove the effect of the approached theory. Figure 8 displays some examples of the original gray images and the corresponding result of the simulation of the threshold segment. The gray images of the road are shown in Figure 8(a), Figure 8(e) and Figure 8(i). Figure 8(b), Figure $8(\mathrm{f})$, Figure $8(\mathrm{j})$ illustrate the binary images which were processed by the iteration threshold algorithm. Figure 8(c), Figure 8(g) and Figure 8(k) display how the OTSU algorithm dealt with the gray images. Figure $8(\mathrm{~d})$, Figure $8(\mathrm{~h})$, Figure $8(\mathrm{l})$ are the binary images which were processed by the watershed algorith$\mathrm{m}$. Comparing the images in Figure 8 which are processed by the three kinds of algorithms of the threshold segment, it was discovered that the lane markings in Figure 8(b), Figure 8(c), Figure 8(f), Figure 8(g), Figure $8(\mathrm{j})$ and Figure $8(\mathrm{k})$ were clear. Thus, the performance of the Iteration threshold algorithm and the OTSU threshold algorithm were better and the performance of the watershed algorithm was slightly worse.

In addition, Table 1 is the time of processing the images by the three algorithms in Figure 8. Comparing the three threshold segmentation algorithms, the OTSU algorithm processed image a bit faster than the Iteration threshold algorithm. It took the longest time to process the image by the watershed algorithm. So in this paper, the OTSU algorithm was used to segment the images after we consider comprehensive.

Figure 9 shows an example of the lane recognition through using Hough Transform algorithm. Figure 9(d) illustrates the Hough matrix and peak points. The HT constructs $\rho-\theta$ Hough space, in which not single 


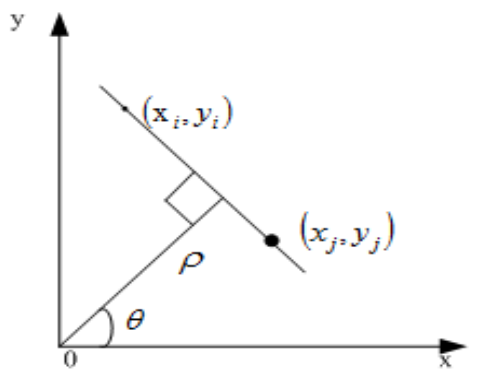

Figure 6 Parameter schematic.

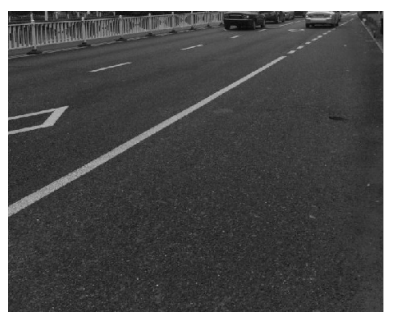

(a) Gray image

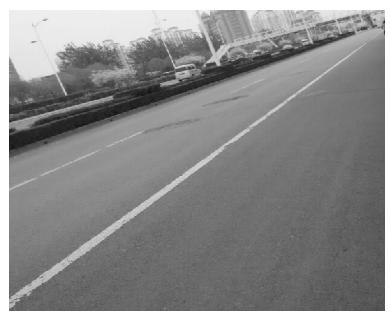

(e) Gray image

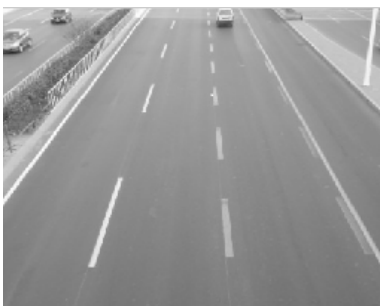

(i) Gray image

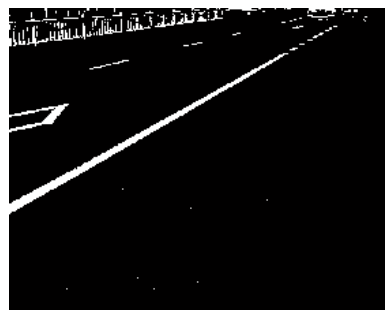

(b) Iteration threshold

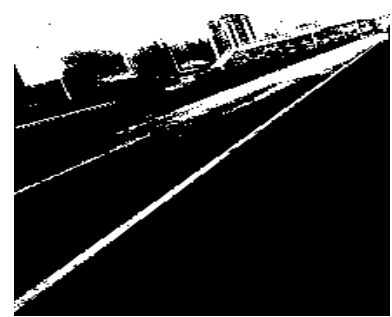

(f) Iteration threshold

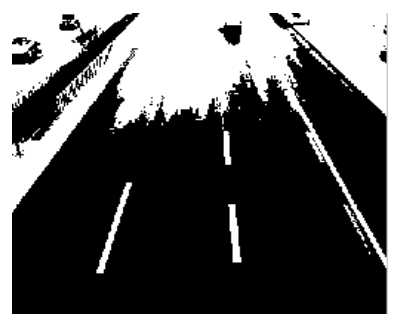

(j) Iteration threshold

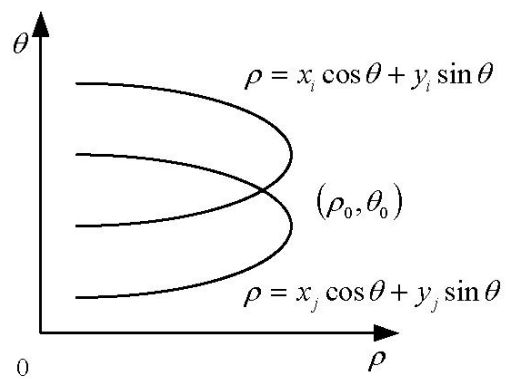

Figure 7 Cartesian coordinate mapped into the parameter space.

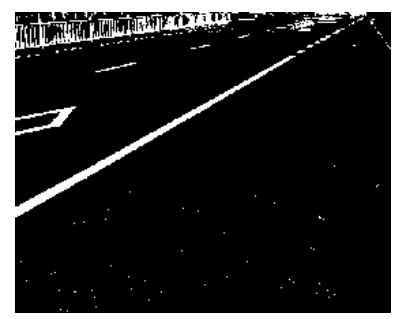

(c) OTSU algorithm

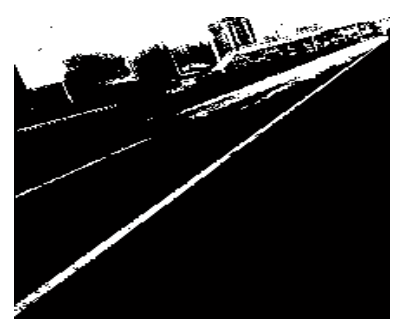

(g) OTSU algorithm

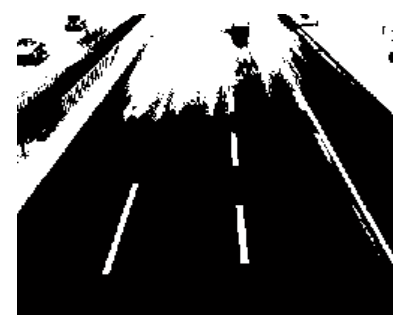

(k) OTSU algorithm

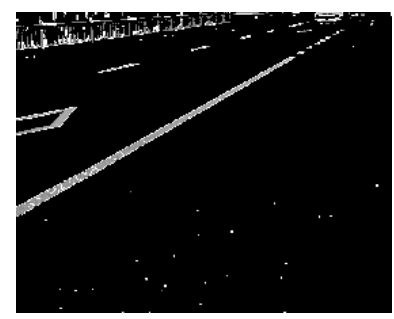

(d) Watershed algorithm

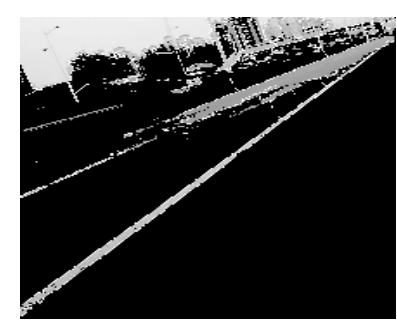

(h) Watershed algorithm

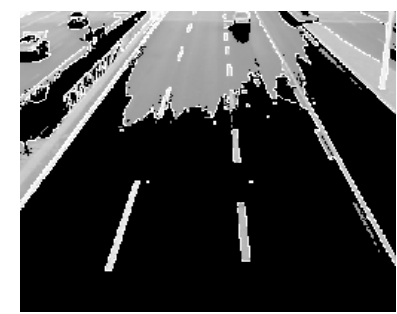

(1) Watershed algorithm

Figure 8 Two Subfigures

global maximum points that may be an outlier but local maximum points through a heuristic approach [8] were searched. For example, the first five peak points whose values are larger than the 0.3 times of the maximum value were selected in the Figure 9(d) and the five points with bright color were marked to identify easily. The number of the intersections in the Figure 9(d) is the number of the lanes according to the Hough transform algorithm.

The recognized lane markings images are displayed in Figure 9(e), Figure 9(f) and Figure 9(g). The threshold value was set to extract the lane lines for $K$. According to the detected lanes images of Figure 9, the threshold $(K)$ to extracting lane lines was higher, and the marked red lines were fewer. At the same time, the irrelevant details could be reduced. On the contrary, the threshold $(\mathrm{K})$ to extracting lines was lower, and the marked red lines were more. Meanwhile, more and more irrelevant details may also be assigned by red. By adjusting the threshold properly, it can make better effect for the straight lines extraction. 
Table 1 Run time of the three algorithms

\begin{tabular}{|c|c|c|c|c|c|c|c|c|c|}
\hline $\begin{array}{c}\text { Segment } \\
\text { algorithms }\end{array}$ & Fig.8b & Fig.8c & Fig.8d & Fig.8f & Fig.8g & Fig.8h & Fig.8j & Fig.8k & Fig.8I \\
\hline Rum time(s) & 0.0370 & 0.0227 & 0.9216 & 0.2262 & 0.0359 & 1.2027 & 0.5210 & 0.5176 & 2.6377 \\
\hline
\end{tabular}

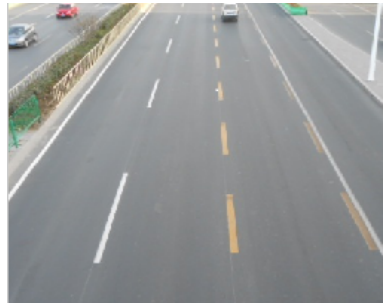

(a) Original image

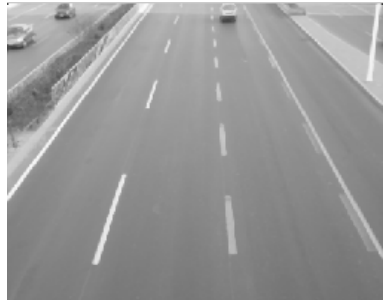

(b) Gray image

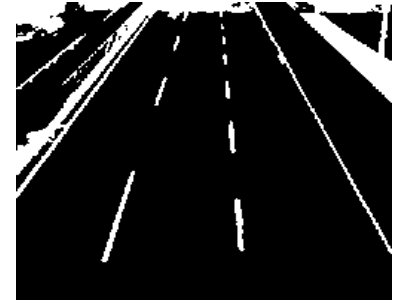

(c) Binary image

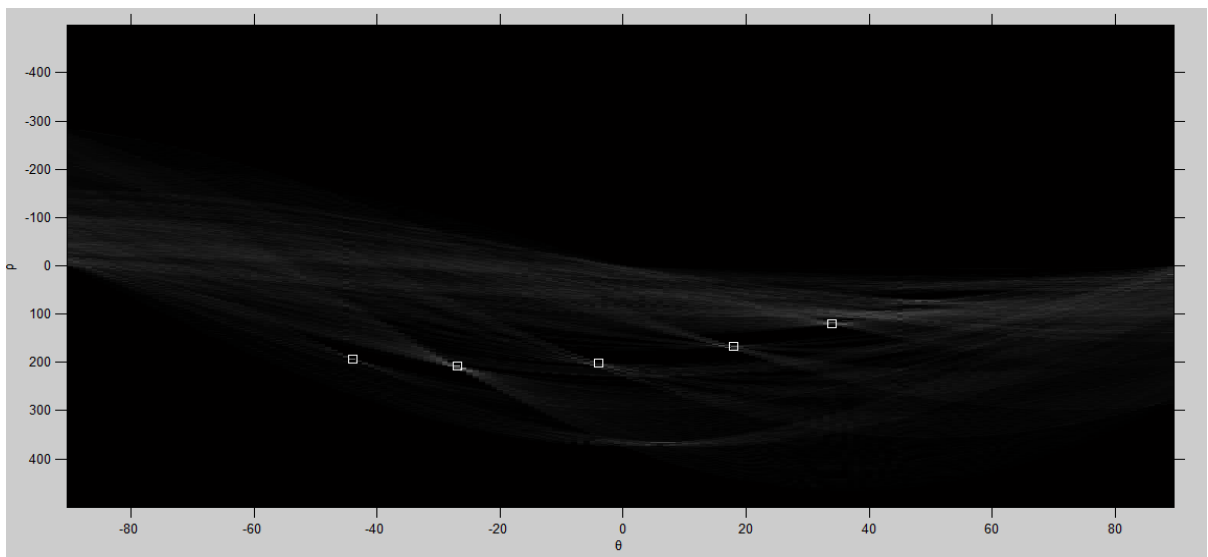

(d) Curve of the image in the parameter space and peak points

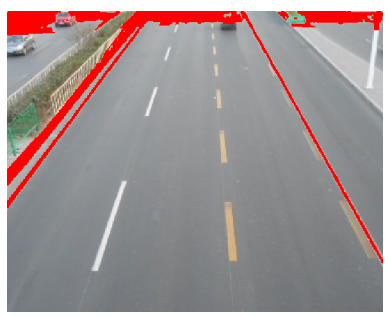

(e) $\mathrm{K}=200$

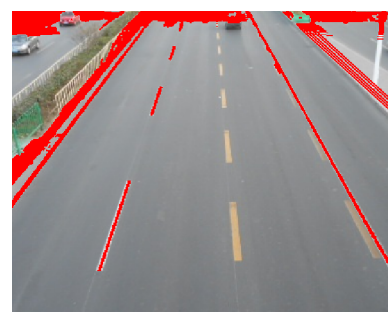

(f) $\mathrm{K}=160$

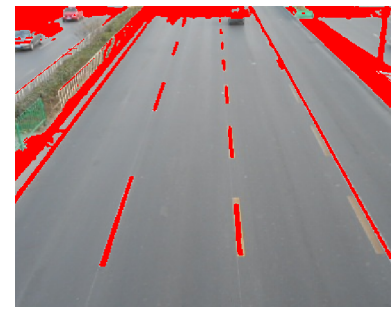

(g) $\mathrm{K}=120$

Figure 9 The processing of the Hough Transform of the road image.

\section{Conclusions and future work}

The proposed system has the ability to handle structured road type using only one camera sensor. In order to be able to achieve efficient effect in lane detection, this paper mainly compared the three kinds of algorithms to threshing the image in the threshold segment process and obtains the good effect of the OTSU algorithm; HT(Hough Transform) algorithm was used to study the lane recognition and the satisfactory results had been obtained. In this way high accuracy with rel- atively simple and efficient lane detection algorithms were achieved. But for the serious interference on the road conditions and the unstructured road was prospered in [9], the accuracy of the lane recognition still needs to be improved and resolved in the following research.

\section{References}

[1] Lee J. W., "A machine vision system for lane departure detection", Computer Vision and Image Under- 
standing, Vol.86, No.1, pp.52-78, 2002.

[2] B. Yu, W. Zhang, Y. Cai, "A lane departure warning system based on machine vision", In: Pacific-Asia Workshop on Computational Intelligence and Industrial Application, Vol.1, pp.197-201, 2008.

[3] D. Ming,Q. Wang,J. Luo, et al. "Evaluation of high spatial resolution remote sensing image segmentation algorithms", In: 2nd International Congress on Image and Signal Processing, pp.1-5, 2005.

[4] A. Borkar, M. Hayes and M.T. Smith, "Robust lane detection and tracking with RANSAC and Kalman filter", In: Proc. Int. Conf. on Image Processing, Cairo, Egypt, Oct., 2009.

[5] Z. Kim, "Robust lane detection and tracking in challenging scenarios", IEEE Trans. Intell. Transp. Syst., pp.16-26, No.9, 2008.

[6] A. Lopez, J. Serrat, F. Lumbreras, et al. "Robust lane markings detection and road geometry computation", International Journal of Automotive Technology, Vol.11, No.3, pp.395-407, 2010.

[7] J. W. Lee, U. K. Yi, "A lane departure identification based on LBPE, Hough Transform and Linear Regression", Computer Vision and Image Understanding, Vol.99, No.3, pp.359-383, 2005.

[8] J. W. Lee, C. D. Kee, U. K. Yi, "A new approach for lane departure identification", In:Proc of IEEE Intelligent Vehicles Symposim, pp.100-105, 2003.

[9] H. Y. Cheng, C. C. Yu, C. C. Tseng, et al. "Environment classification and hierarchical lane detection for structured and unstructured roads", IET Computer Vision, Vol.4, No.1, pp.37-49, 2010. 